\title{
Controlled Oral Word Association Test
}

National Cancer Institute

\section{Source}

National Cancer Institute. Controlled Oral Word Association Test. NCI Thesaurus. Code C114144.

A test that measures the verbal fluency of an individual. This tool measures both semantic fluency and phonetic fluency. 